\title{
A review of mobile applications to help adolescent and young adult cancer patients
}

This article was published in the following Dove Press journal:

Adolescent Health, Medicine and Therapeutics

18 August 2015

Number of times this article has been viewed

\author{
Kimberly M Wesley' \\ Philip J Fizur ${ }^{2}$ \\ 'Department of Psychology, \\ St Jude Children's Research Hospital, \\ Memphis, TN, ${ }^{2}$ Department of \\ Psychology, La Salle University, \\ Philadelphia, PA, USA
}

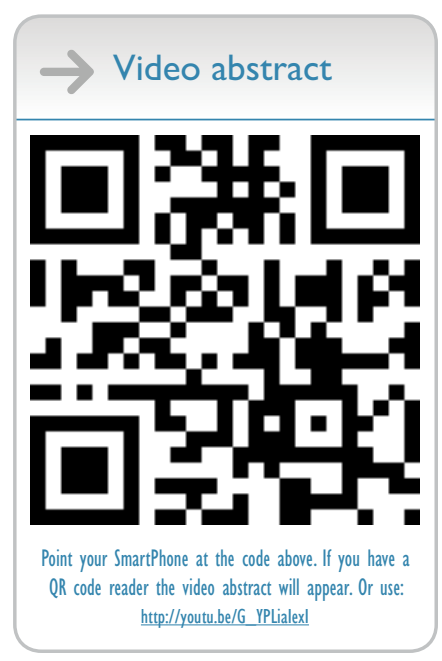

Correspondence: Kimberly M Wesley Department of Psychology, St Jude Children's Research Hospital,

262 Danny Thomas Place - mail stop 740, Memphis, TN 38105, USA Tel + I 90I 5957764

Email kimberlywesleypsyd@gmail.com
Objective: To review research articles utilizing mobile applications with adolescent and young adult (AYA) cancer patients.

Materials and methods: We identified articles via online searches and reference lists (eg, PsycInfo, PubMed). Articles were reviewed by two study team members for target population, stated purpose, technological utilization, sample size, demographic characteristics, and outcome data. Strengths and weaknesses of each study were described.

Results: Of 19 identified manuscripts, six met full inclusion criteria for this review (four smartphone applications and two tablet applications). One additional article that included an application not specific to oncology but included AYA patients with cancer within the target sample was also reviewed. Uses of these applications included symptom tracking, pain management, monitoring of eating habits following bone marrow transplant, monitoring of mucositis, and improving medication management. Utility results from pilot studies are presented.

Conclusion: Mobile applications are growing in number and increasingly available to AYAs with and without chronic illness. These applications may prove useful in helping to support AYAs throughout their cancer treatment and beyond. However, few applications provide empirical data supporting their utility. Numerous strengths and benefits of these applications include increased accessibility to educational resources and self-management strategies, more frequent physical and emotional symptom tracking, and increased access to peer support. Despite these strengths, numerous limitations are identified, highlighting the need for future research in this area.

Keywords: adolescent, young adult, cancer, smartphone, mobile, applications

\section{Background}

Each year, nearly 70,000 adolescents and young adults (AYAs) are diagnosed with cancer in the US. ${ }^{1}$ A cancer diagnosis during this time period disrupts the achievement of developmental milestones, such as increasing independence and career/educational goal attainment, and also interferes with social relationships. ${ }^{2,3}$ Cancer treatments may cause patients to experience a range of physical and psychological side effects, including pain, fatigue, nausea, vomiting, appetite disruption, weight changes, body-image disruption (eg, scars, alopecia, amputation), seizures, mucositis, depression, anxiety, and increased negative affect. ${ }^{4,5}$ Socially, medical treatment may require isolation from peers ${ }^{4}$ and frequent school absences. ${ }^{6}$ While a number of these concerns can be addressed through psychosocial care, one study found that $41 \%$ of AYA patients felt as though their service needs were not met, with $75 \%$ reporting that they wanted to be involved in a support group but were not connected with an appropriate resource. ${ }^{2}$ Additionally, over half of those participants reported wanting to have more information 
about their disease and treatment, which was not provided to them. Potential reasons for this poor provision of resources include lack of insurance and financial barriers to care, and lack of available and qualified medical and psychosocial care providers. Electronic interventions may address some of these concerns, as they are generally more accessible, more easily updated, and can be cost-effective. ${ }^{7}$

Therefore, a potential method for providing access to resources for AYAs diagnosed with cancer may involve mobile technology. An increasing number of AYAs have access to mobile technology. Recently, it was estimated that $75 \%$ of adolescents between the ages of 12 and 17 years own a mobile phone, with approximately $67 \%$ owning an iPhone..$^{8,9}$ Nearly $91 \%$ of teenagers report having access to the Internet through the use of smartphones, tablets, and other mobile devices. ${ }^{10}$ Given the high level of accessibility, it is natural that mobile applications geared toward AYAs with specific medical conditions have been developed and are beginning to be used in clinical research studies.

Numerous purposes of mobile applications have been identified in the literature. One goal of smartphone applications for oncology patients is to improve accuracy of symptom tracking, as these applications are readily accessible so that patients can report their symptoms in the moment, rather than having to attempt to remember these symptoms at a later time. ${ }^{11} \mathrm{~A}$ second purpose is to monitor quality of life. ${ }^{12}$ Another goal of smartphone applications is to provide support and feedback to patients in the moment without the patient being physically present in a medical setting, such as offering an intervention or an alert being sent to a patient to seek medical care after reporting a specific symptom. ${ }^{13}$ Additionally, mobile technology has been designed to facilitate social networking with patients experiencing similar medical conditions and treatments to increase social support and shared resources. ${ }^{14}$ Another advantage is that AYAs may feel more comfortable sharing sensitive information through electronic means, thereby increasing the amount of symptoms or other relevant information they share with their medical providers and interdisciplinary team. ${ }^{15}$

Smartphone and tablet applications have been used with a variety of other medical conditions to help facilitate healthbehavior change. For example, mobile applications can also be used to monitor and improve medication adherence by providing daily logs and reminders. ${ }^{16}$ Additional applications are available to assist with smoking cessation. ${ }^{17}$ Smartphone and tablet applications are also available to aid in weight management through various means, including monitoring physical activity ${ }^{18}$ and recording dietary intake. ${ }^{19}$
A large number of smartphone applications are currently available to be downloaded. For example, Pandey et al identified 77 oncology-related applications geared toward either medical providers or lay populations through the Apple iTunes store during a search conducted in mid-2011. ${ }^{20}$ The authors noted their concern about the lack of specificity and validity of the applications, which could endanger the safety of patients and others using the applications. Another review identified 295 smartphone applications related to cancer, including applications designed to promote cancer awareness, support disease management, and increase social support. ${ }^{21}$ The authors noted that none of these applications provided empirical support or evaluative data in the descriptions of the applications, which was a significant limitation of the applications included in this review. More recently, another review located nearly 300 patient-focused pain-management applications available to consumers, though these applications had not been thoroughly tested or empirically supported. ${ }^{22}$ These authors noted the importance of carefully reviewing these applications and involving medical providers in the development of these tools.

Previous research has identified hundreds of applications that may prove to be useful to AYA cancer patients. However, a small proportion of these applications have been examined empirically, with an even smaller percentage tested with AYAs diagnosed with cancer. The purpose of this review was to identify mobile applications for AYAs diagnosed with cancer that have evidence on their effectiveness and/or usability. The identified research question was: "What mobile applications have been identified as potentially helpful to adolescents and young adults diagnosed with cancer and have been published in peer-reviewed journals?" A discussion of the strengths of these applications, limitations, and future directions is provided.

\section{Materials and methods}

The methodology for this review was based on Arksey and O'Malley's framework for scoping reviews. ${ }^{23}$ The steps outlined in this framework include to 1) identify the broader research question, 2) conduct a comprehensive identification of relevant research studies, 3) select studies following the establishment of inclusion/exclusion criteria, 4) chart the data, and 5) summarize and report the results. A final step includes consultation with key stakeholders, which is outside the scope of this review; therefore, this step was not completed.

\section{Procedure}

Multiple academic databases (PsycInfo, PubMed, Ovid, Scopus, ProQuest Health and Medicine) and general online 
search engines (Google Scholar) were searched four times by both authors between October 2014 and March 2015 to identify smartphone and tablet applications designed for AYA cancer patients. Combinations of the following search terms were used in searches: "adolescent", "young adult", "AYA", "oncology", "cancer", "smartphone", "mobile phone", "cellular phone", "iPad”, "iPhone”, "Android”, “iOS”, "app store", "market place", "application", and "app".

\section{Review and description of technology}

In order to be included in this review, the manuscripts were required to 1) include a specific mobile application, 2) include adolescent and/or young adult cancer patients (age 13-39 years) in the pilot study or description of the application, and 3) come from a manuscript that was published in English. Exclusion criteria were 1) lack of a mobile application, 2) oncology patients not included in the pilot study, 3) sample including patients outside of the designated age range, and 4) manuscripts that were not published in English. Overall, 19 manuscripts were identified (see Figure 1 for flowchart). Abstracts were reviewed by both authors, who reached consensus on those abstracts that met the inclusion criteria after each search. This resulted in 12 manuscripts being excluded from this review, as they either did not utilize an oncology population (two manuscripts), included only older adults in the study (five manuscripts), or did not include a smartphone or tablet application (five manuscripts). Of the published manuscripts included in this review, four included a specific smartphone application for use with AYAs with cancer, two included an application for use with this population on a tablet (eg, iPad), and one application included AYA cancer patients in the pilot sample, though the application was not developed specifically for this demographic.

\section{Results Smartphone applications}

Four manuscripts identified during this review included a smartphone application pilot tested specifically with AYA cancer patients (Table 1).

\section{Cherry}

Cherry is a mobile application developed for the Android operating system. ${ }^{24}$ Cherry was designed to provide children and adolescents with cancer with a diary tool to monitor their

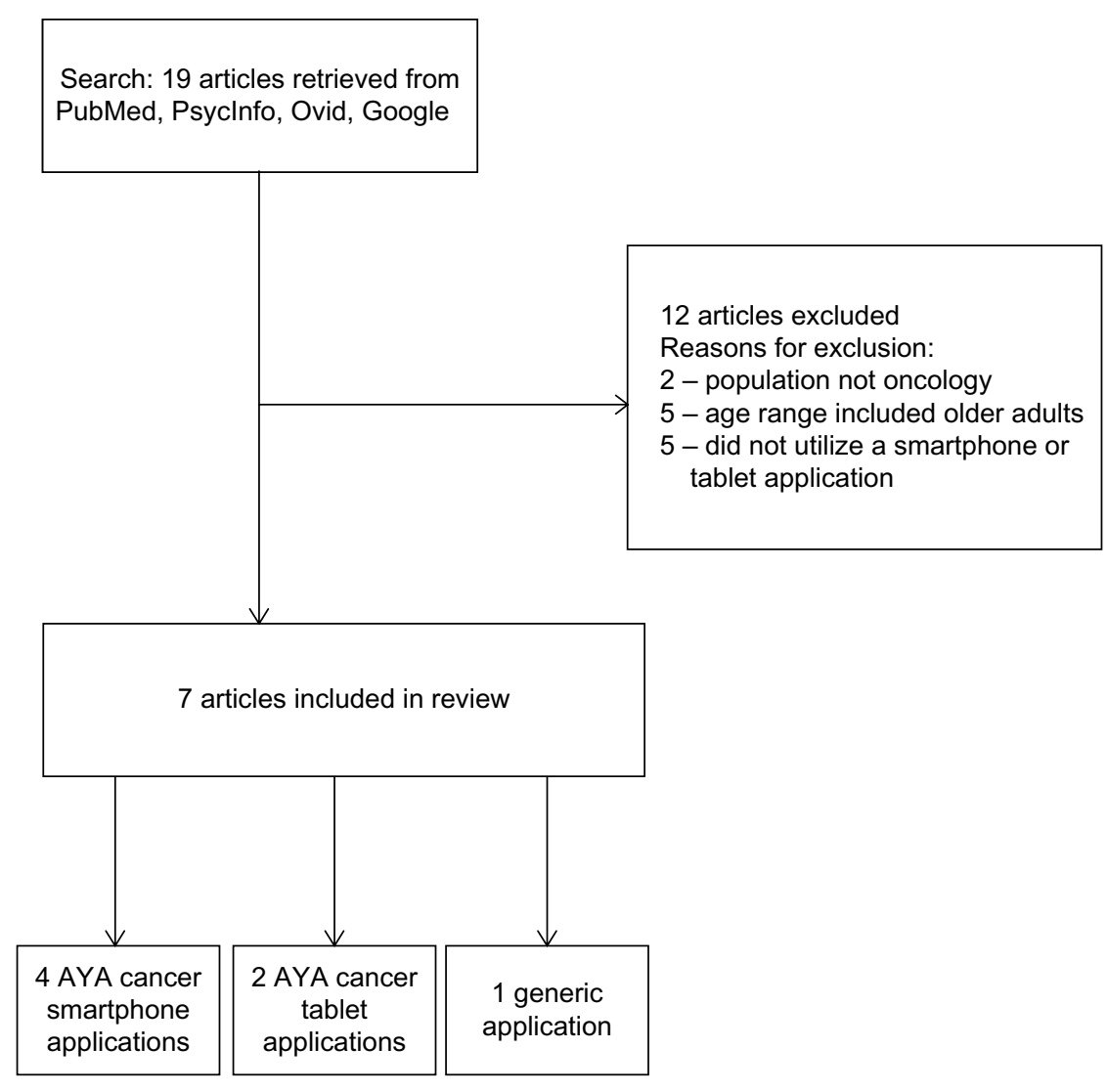

Figure I Flowchart.

Abbreviation: AYA, adolescent and young adult. 
Table I Smartphone applications

\begin{tabular}{|c|c|c|c|c|}
\hline Study & Sample & Application & Platform & Purpose \\
\hline Baggott et al ${ }^{15}$ & $\begin{array}{l}\text { Ten oncology patients } \\
\text { (mean age } 18.2 \text { years) }\end{array}$ & $\begin{array}{l}\text { Mobile Oncology Symptom } \\
\text { Tracker (mOST) }\end{array}$ & Apple iOS & Record medical symptoms \\
\hline Berntsen and Babic ${ }^{24}$ & - & Cherry & Android & $\begin{array}{l}\text { Diary to share with doctor, friends, and } \\
\text { parents; provides disease information } \\
\text { and feedback from physicians }\end{array}$ \\
\hline Rodgers et $\mathrm{al}^{25}$ & $\begin{array}{l}16 \text { adolescents } \\
\text { (age } 11-18 \text { years) }\end{array}$ & Eating after Transplant (EAT!) & Not specified & $\begin{array}{l}\text { Increase self-management of eating- } \\
\text { related issues during hematopoietic } \\
\text { stem cell transplantation recovery }\end{array}$ \\
\hline Stinson et $\mathrm{a}^{\prime \prime}$ & $\begin{array}{l}\text { I4 patients with cancer } \\
\text { (mean age } 13.2 \text { years) }\end{array}$ & Pain Squad & Apple iOS & $\begin{array}{l}\text { Track pain (eg, intensity, location) } \\
\text { and intervention (eg, medication, } \\
\text { nonpharmacologic strategies) }\end{array}$ \\
\hline
\end{tabular}

experiences and to share the diary entries with their parents, peers, or providers. Additional functions include access to disease-related information and the ability to receive feedback from physicians. Pilot data were not available at the time of this review, though the authors stated that a preliminary evaluation demonstrated enthusiasm and easiness of use.

\section{Eating after Transplant}

Eating after Transplant (EAT!) is an application developed for use on smartphones. ${ }^{25}$ EAT! was designed to provide information to adolescents during the recovery period after hematopoietic stem cell transplantation. This application provides information about symptoms and self-management strategies. EAT! focuses on seven aspects of eating during the acute recovery period: appetite, food choice, control of eating, nausea and vomiting, taste changes, dry mouth, and normalcy. Sixteen adolescents (age 11-18 years, mean age not available) participated in the pilot study. Acceptability was rated at three time points ( 20 days, 40 days, and 60 days after hospital discharge) using six questions related to readability, quickness of use, enjoyment, helpfulness, ease of use, and program recommendation on a 5-point Likert scale. Usability was rated using three questions related to time needed to locate information, estimated time of use, and number of topics reviewed in the program over 20 days. Usability was measured using the amount of time recorded using the application via a tracking device in the application. High acceptability $(28.5 / 30,28.9 / 30$, and 28.8/30 at each respective time point) and usability (11.2/15, 10.1/15, and $10.3 / 15$, respectively) were reported by the participants. Participants used the application for the first 100 days posttransplant; use of the application decreased throughout the course of the study, from 8.6 minutes per day within the first 20 days to 0.6 minutes per day during the final 20 days of the study.

\section{Mobile Oncology Symptom Tracker}

Baggott et al presented Mobile Oncology Symptom Tracker (mOST), an application available for iPhone. ${ }^{15}$ The application was used daily to assess the severity of disease or treatment-related symptoms (ie, pain, nausea, fatigue, vomiting, and sleep quality), as well as the occurrence of other symptoms (eg, diarrhea, fever, mouth sores, and dizziness). Finally, patients were asked to describe their current mood. The authors utilized validated measures, including the Color Analog Scale, Pediatric Nausea Assessment Tool, and Faces Pain Scale-Revised to assess symptoms. A postuse interview was conducted at the end of the study. mOST was pilot tested with ten AYAs with various cancer diagnoses (mean age 18.2 years, standard deviation [SD] 2.9 years) over 3 weeks. Participants were instructed to report their symptoms each day, and could program two customizable reminder messages to facilitate compliance. Overall compliance was $91 \%$, and adherence rates did not differ among the 3 weeks of the pilot study $(F=1.016, P=0.38)$. Following completion of the study, $100 \%$ of the users reported that the application was easy to use, and selected positive descriptors (eg, rewarding, educational, interesting) to describe the application.

\section{Pain Squad}

Pain Squad is an application available on iPhone. ${ }^{11}$ Pain Squad serves as a pain diary that prompts patients to track their daily pain intensity, duration, location, and the impact of pain on daily functioning. This application also allows the user to log medications and other pain-management strategies that have been used. Pain Squad has been pilot tested with a sample of 14 adolescents (mean age 13.2 years, SD 2.3 years) diagnosed with various forms of cancer, who were asked to complete pain assessments twice daily for 14 days. Participants completed an evaluation questionnaire (using a 4-point Likert scale) after this 2-week period. The mean 
compliance rate for using the application twice daily was $81 \%$ (SD 22\%), and $86 \%$ of the adolescents reported liking the application, with $79 \%$ reporting that the application was easy to use. Pain Squad uses game-based features and virtual rewards to increase appeal to adolescents.

\section{Tablet applications}

Two manuscripts identified during this review included a tablet application pilot tested specifically with AYA cancer patients (Table 2).

\section{Children's International Mucositis Evaluation \\ Scale - electronic version}

Children's International Mucositis Evaluation Scale electronic version (eChIMES) is an application available on the iPad. ${ }^{26}$ This application is an electronic version of a previously validated paper measure - ChIMES. eChIMES measures various symptoms associated with mucositis (eg, pain, difficulty swallowing, appearance of mouth sores) and tracks the use of medication for pain management. eChIMES was pilot tested with a sample of 40 children and adolescents (median age 12.4 years, range $8.0-17.8$ years) currently receiving treatment for cancer. At the end of the study, the authors utilized a semistructured interview and questionnaire with a 5-point Likert scale to measure usability and acceptability. All participants rated this application as easy to use, and most (95\%) said it was easy to understand, with $92 \%$ reporting that this application was a suitable way to measure mucositis.

\section{Computerized Symptom Capture Tool}

Computerized Symptom Capture Tool (C-SCAT) is an application developed for the iPad. ${ }^{27}$ This application features 30 symptoms that are commonly experienced by AYAs with cancer, and also gathers information regarding possible causes of the symptoms, alleviating/exacerbating factors, self-management strategies that were used in attempt to manage the symptom, and the effect of the symptoms on daily activities. Additionally, the respondent can cluster related symptoms together. C-SCAT was pilot tested with a sample of 40 adolescents (median age 15 years, range 13-18 years) and 32 young adults (median age 21.5 years, range 19-29 years) receiving chemotherapy. Participants completed C-SCAT 24-96 hours after receiving an initial dose of a chemotherapy cycle. Overall, participants rated this application as clear (89\%), with easy-to-follow instructions (100\%), easy to complete (94\%), and useful (87\%). All participants completed C-SCAT, with 74\% (95\% confidence interval $62 \%-83 \%$ ) reporting that the output was an accurate representation of their symptoms.

\section{Applications not specific to cancer}

One application identified during this review includes patients diagnosed with a variety of chronic illnesses, including patients with cancer within the designated age range (13-39 years) (Table 3).

\section{Medication Plan (Medikamentenplan)}

Medication Plan was created as a smartphone application on the Apple iOS platform. ${ }^{28}$ The application consists of features allowing users to customize their medication plans to their own personal needs and prescriptions. For example, users are allowed to input their medication dosage, duration of treatment, how often to take the medication, what times to take their medication, and other special instructions (eg, take with food or take on an empty stomach). Data were collected from 11,688 smartphone users 6-87 years old (mean age 44 years), and $9 \%$ reported suffering from cancer. Other diagnoses included but were not limited to cardiovascular diseases, diabetes, and lung disease. Feedback indicated some difficulties with software bugs, but otherwise this application was generally well received. Regular use of the application decreased after 2 months.

\section{Discussion}

With increased prevalence of smartphones and tablets, there has been an increase in the number of applications specific to chronic medical conditions. This review identified

Table 2 Tablet applications

\begin{tabular}{|c|c|c|c|c|}
\hline Study & Sample & Application & Platform & Purpose \\
\hline $\begin{array}{l}\text { Macpherson } \\
\text { et } \mathrm{al}^{27}\end{array}$ & $\begin{array}{l}40 \text { adolescents (median age } \\
\text { I } 5 \text { years) and } 32 \text { young adults } \\
\text { (median age } 2 I .5 \text { years) } \\
\text { receiving chemotherapy }\end{array}$ & $\begin{array}{l}\text { Computerized Symptom } \\
\text { Capture Tool (C-SCAT) }\end{array}$ & Apple iOS & $\begin{array}{l}\text { Measure of various treatment- } \\
\text { related symptoms, potential } \\
\text { causes, and attempted self- } \\
\text { management strategies }\end{array}$ \\
\hline $\begin{array}{l}\text { Tomlinson } \\
\text { et } \mathrm{al}^{26}\end{array}$ & $\begin{array}{l}40 \text { children and adolescents } \\
\text { receiving active treatment for } \\
\text { cancer (median age } 12.4 \text { years) }\end{array}$ & $\begin{array}{l}\text { Children's International } \\
\text { Mucositis Evaluation Scale - } \\
\text { electronic version (eChIMES) }\end{array}$ & Apple iOS & Measure of oral mucositis \\
\hline
\end{tabular}


Table 3 Applications not specific to cancer

\begin{tabular}{|c|c|c|c|c|}
\hline Study & Sample & Application & Platform & Purpose \\
\hline $\begin{array}{l}\text { Becker } \\
\text { et } \mathrm{al}^{28}\end{array}$ & $\begin{array}{l}\text { I I,688 smartphone users (age 6-87 [mean 44] } \\
\text { years; } 205 \text { [9\%] diagnosed with cancer) }\end{array}$ & $\begin{array}{l}\text { Medication Plan } \\
\text { (Medikamentenplan) }\end{array}$ & Apple iOS & $\begin{array}{l}\text { Designed to maintain medication } \\
\text { list and improve compliance }\end{array}$ \\
\hline
\end{tabular}

several smartphone and tablet applications geared toward AYAs diagnosed with cancer. These applications include a variety of topics, including monitoring general symptoms (eg, mOST) ${ }^{15}$ as well as more specific side effects, such as mucositis, eating-related issues following hematopoietic stem cell transplantation, and pain. ${ }^{11}$ Additional applications include diaries to keep track of physical and emotional responses, and some provide feedback from the application or from medical staff to help identify coping strategies for the symptoms that were reported at a given time (eg, Cherry). ${ }^{24}$ One application also provides tools to assist with issues to improve medication adherence, including tools to help schedule dosages and keep track of special instructions. ${ }^{28}$ This particular application is highly customizable to the specific needs of each individual. The services offered by these applications can be used to address concerns raised by the AYAs surveyed by the AYA Hope Collaborative Study Group, who reported a lack of support and education during their cancer treatment. ${ }^{2}$ Specifically, mobile applications are available to provide disease-related information, facilitate communication with medical providers, and provide recommendations for pain-management strategies. If medical providers and psychosocial team members increase awareness of these pilot-tested applications, they can recommend use to clinically relevant patients. However, none of the studies identified in this review was specific to increasing access to social support or addressing other behavioral health concerns (eg, sleep, weight management, smoking). Further, several applications were identified that have been used with multiple chronic health populations, but did not have specific data supporting efficacy for oncology populations (eg, iCanCope with Pain) ${ }^{29}$ Therefore, other applications are likely available to help AYA patients diagnosed with cancer, though the evidence supporting the use of these applications is not yet available.

Many potential limitations of the aforementioned pilot studies and use of mobile technology were apparent throughout this review. The electronic devices used in these studies can be very expensive, and damage to these devices can be costly. ${ }^{26}$ Though an increasing number of AYAs have access to smartphones and tablets, approximately $25 \%$ do not have regular access to the Internet or mobile applications. ${ }^{10}$ Therefore, while some research studies provided smartphones with Internet access to participants (eg, mOST),${ }^{15}$ this is not possible for all oncology patients. Of note, individuals of lower socioeconomic status with fewer financial resources may thus have less access to smartphones and tablets, thereby limiting their access to potentially helpful mobile applications. These individuals may especially benefit from mobile resources, as numerous barriers prevent patients from lower sociodemographic backgrounds from accessing information and support through other means (eg, lack of insurance, lack of transportation). However, the advent of "pay-as-you-go" smartphones may allow a wider population to utilize this technology. ${ }^{30}$

Limitations within the individual participants are notable. The participants identified several barriers to using the applications consistently, including forgetting the smartphone and thus being unable to make an entry, ${ }^{11}$ forgetting to make entries, and feeling too sick to make entries. ${ }^{15}$ Emergency medical treatment sometimes prevented participants from making entries. ${ }^{11}$ These intraindividual characteristics highlight several important barriers to effective use of these applications, thereby limiting the utility of this technology. The small pilot studies may have included highly motivated individuals who were willing to utilize the applications and may have been more willing to say they were useful and user-friendly, thereby inflating both the compliance and usability ratings. Therefore, it is unclear how well these applications will generalize to other populations. While most of the studies presented a very high response rate, many of these trials took place over small periods of time (eg, 2 weeks), which may have also inflated the reported compliance rates. The EAT! trial lasted for the first 100 days posttransplant, and saw a decrease in use of the application over time. ${ }^{25}$ Therefore, it is unclear how useful these applications will be for long-term use. Another concern is in regard to publication bias, which may have prevented additional applications from surfacing in the existing literature base. Additionally, the search terms listed earlier may have failed to identify published manuscripts, thereby resulting in an incomplete retrieval of the extant research.

Finally, limitations in regard to the applications themselves warrant discussion. First, AYAs are a very heterogeneous group, and large variability exists in regard to the developmental level of patients in this group (ie, variability between a 13 -year-old patient compared to a 25 -year-old patient). 
Therefore, it is very difficult to create specific applications that cater to the various developmental levels of younger AYAs. ${ }^{27}$ Another potential limitation is the specific nature of some applications. For example, the eChIMES application presented by Tomlinson et al focused solely on mucositis. ${ }^{26}$ While this is an important means of assessing symptoms experienced by patients suffering from mucositis, this application is not useful for individuals without mucositis. Therefore, such applications as this may be expanded to include other symptoms in order to be useful for a larger population.

Future directions in research may include a closer evaluation of many of these applications with AYA oncology patients, as well as examining a wider scope of services and interventions offered through these applications (eg, including applications that involve greater social interaction and peer support for patients). Additionally, to address several of the key limitations of the existing research, these studies should include larger sample sizes with intervention periods of longer duration. Further, given the limits of patient selfreport of physical symptoms, attention should be paid to integrating new technologies that connect to these applications to report vital statistics (eg, the TempTraq wearable Bluetooth thermometer to keep clinicians abreast of fevers that could indicate medical complications). As noted earlier, additional factors, such as sociodemographic status, may affect accessibility to mobile applications. The impact of these factors can be a subject of future research.

In conclusion, smartphone and tablet applications are growing in number, and may provide access to resources to help improve quality of life and address unmet needs of AYAs diagnosed with cancer. While few applications have been empirically evaluated with this population, the applications presented in this review have received favorable ratings in regard to usability and utility. This review highlights the need for respected authorities to create and research applications that may benefit cancer patients, to ensure accuracy of information, utility of the applications, and developmental appropriateness for the targeted population. This is a promising direction for collaborative research with the goal of improving the quality of life and overall functioning of AYA cancer patients.

\section{Disclosure}

The authors report no conflicts of interest in this work.

\section{References}

1. National Cancer Institute. A snapshot of adolescent and young adult cancers. 2014. Available from: http://www.cancer.gov/research/progress/ snapshots/adolescent-young-adult. Accessed May 19, 2015.
2. Keegan TH, Lichtensztajn DY, Kato I, et al. Unmet adolescent and young adult cancer survivors information and service needs: a populationbased cancer registry study. J Cancer Surviv. 2012;6:239-250.

3. Shama W, Lucchetta S. Psychosocial issues of the adolescent cancer patient and the development of the Teenage Outreach Program (TOP). J Psychosoc Oncol. 2007;25:99-112.

4. Ruland C, Hamilton G, Schjødt-Osmo B. The complexity of symptoms and problems experienced in children with cancer: a review of the literature. J Pain Symptom Manage. 2009;37:403-418.

5. Wesley KM, Zelikovsky N, Schwartz LA. Physical symptoms, perceived social support, and affect in adolescents with cancer. $J$ Psychosoc Oncol. 2013;31:451-467.

6. Cavusoglu H. Problems related to the diagnosis and treatment of adolescents with leukemia. Issues Compr Pediatr Nurs. 2000;23:15-26.

7. Beale IL, Kato PM, Marin-Bowling VM, Guthrie N, Cole SW. Improvement in cancer-related knowledge following use of a psychoeducational video game for adolescents and young adults with cancer. J Adolesc Health. 2007;41:263-270.

8. Lenhart A, Ling R, Campbell S, Purcell K. Teens and mobile phones. 2010. Available from: http://pewinternet.org/Reports/2010/Teens-andMobile-Phones.aspx. Accessed February 4, 2015.

9. Piper Jaffray Companies. Taking stock with teens Fall 2014. 2014. Available from: http://www.piperjaffray.com/3col.aspx?id=3268. Accessed July 14, 2015.

10. Lenhart A. Mobile access shifts social media use and other online activities. Available from: http://www.pewinternet.org/2015/04/09/mobile-accessshifts-social-media-use-and-other-online-activities/. Accessed July 14, 2015.

11. Stinson JN, Jibb LA, Nguyen C, et al. Development and testing of a multidimensional iPhone pain assessment application for adolescents with cancer. $J$ Med Internet Res. 2013;15:e51.

12. Wu W, Johnson R, Schepp KG, Berry DL. Electronic self-report symptom and quality of life for adolescent patients with cancer. Cancer Nurs. 2011;34:479-486.

13. Jibb LA, Stevens BJ, Nathan PC, Seto E, Cafazzo JA, Stinson JN. A smartphone-based pain management app for adolescents with cancer: establishing system requirements and a pain care algorithm based on literature review, interviews, and consensus. JMIR Res Protoc. 2014;3:e15.

14. McLaughlin M, Nam Y, Gould J, et al. A videosharing social networking intervention for young adult cancer survivors. Comput Human Behav. 2012;28:631-641.

15. Baggott C, Gibson F, Coll B, Kletter R, Zeltzer P, Miaskowski C. Initial evaluation of an electronic symptom diary for adolescents with cancer. JMIR Res Protoc. 2012;1:e23.

16. Dayer L, Heldenbrand S, Anderson P, Gubbins PO, Martin BC. Smartphone medication adherence apps: potential benefits to patients and providers. J Am Pharm Assoc. 2013;53:172-181.

17. Ubhi HK, Michie S, Kotz D, Wong WC, West R. A mobile app to aid smoking cessation: preliminary evaluation of SmokeFree28. J Med Internet Res. 2015;17:e17.

18. Yang CH, Maher JP, Conroy DE. Implementation of behavior change techniques in mobile applications for physical activity. Am J Prev Med. 2015;48:452-455.

19. Welch JL, Astroth KS, Perkins SM, et al. Using a mobile application to self-monitor diet and fluid intake among adults receiving hemodialysis. Res Nurs Health. 2013;36:284-298.

20. Pandey A, Hasan S, Dubey D, Sarangi S. Smartphone apps as a source of cancer information: changing trends in health information-seeking behavior. J Cancer Educ. 2013;28:138-142.

21. Bender JL, Yue RY, To MJ, Deacken L, Jadad AR. A lot of action, but not in the right direction: systematic review and content analysis of smartphone applications for the prevention, detection, and management of cancer. J Med Internet Res. 2013;15:e287.

22. Lalloo C, Jibb LA, Rivera J, Agarwal A, Stinson JN. "There's a pain app for that": review of patient-targeted smartphone applications for pain management. Clin J Pain. 2015;31:557-563. 
23. Arksey H, O’Malley L. Scoping studies: towards a methodological framework. Int J Soc Res Methodol. 2005;8:19-32.

24. Berntsen E, Babic A. Cherry: mobile application for children with cancer. Stud Health Technol Inform. 2013;192:1168.

25. Rodgers CC, Krance R, Street RL, Hockenberry MJ. Feasibility of a symptom management intervention for adolescents recovering from a hematopoietic stem cell transplant. Cancer Nurs. 2013;36:394-399.

26. Tomlinson D, Hesser T, Maloney A, Ross S, Naqvi A, Sung L. Development and initial evaluation of electronic Children's International Mucositis Evaluation Scale (eChIMES) for children with cancer. Support Care Cancer. 2013;22:115-119.

27. Macpherson CF, Linder LA, Ameringer S, Erickson J, Stegenga K, Woods NF. Feasibility and acceptability of an iPad application to explore symptom clusters in adolescents and young adults with cancer. Pediatr Blood Cancer. 2014;61:1996-2003.
28. Becker S, Kribben A, Meister S, Diamantidis CJ, Unger N, Mitchell A. User profiles of a smartphone application to support drug adherence: experiences from the iNephro Project. PLoS One. 2013;8:e78547.

29. Stinson, JN, Lalloo C, Harris L, et al. iCanCope with Pain ${ }^{\mathrm{TM}}$ : usercentred design of a web- and mobile-based self-management program for youth with chronic pain based on identified health care needs. Pain Res Manag. 2014;19:257-265.

30. Brown K, Campbell SW, Ling R. Mobile phones bridging the digital divide for teens in the US? Future Internet. 2011;3:144-158.

\section{Publish your work in this journal}

Adolescent Health, Medicine and Therapeutics is an international, peer-reviewed, open access journal focusing on health, pathology, and treatment issues specific to the adolescent age group. All aspects of health maintenance, preventative measures and disease treatment interventions are addressed within the journal and practitioners from all disciplines are invited to submit their work as well as healthcare researchers and patient support groups.. The manuscript management system is completely online and includes a very quick and fair peerreview system. Visit http://www.dovepress.com/testimonials.php to read real quotes from published authors.

Submit your manuscript here: http://www.dovepress.com/adolescent-health-medicine-and-therapeutics-journal 